\title{
Updates to the MSFC Meteoroid Stream Model
}

D. E. Moser and W. J. Cooke (NASA Meteoroid Environment Office, Marshall Space Flight Center)

ABSTRACT The Marshall Space Flight Center (MSFC) Meteoroid Stream Model simulates particle ejection and subsequent evolution from comets in order to provide meteor shower forecasts to spacecraft operators for hazard mitigation and planning purposes. The model, previously detailed in Moser \& Cooke (2004), has recently been updated; the changes include the implementation of the RADAU integrator, an improved planetary treatment, and the inclusion of general relativistic effects in the force function. The results of these updates are investigated with respect to various meteoroid streams and the outcome presented.

\section{BACKGROUND}

What Model of particle ejection and subsequent evolution from comets known for producing meteor showers at Earth.

Why To provide accurate meteor shower forecasts to spacecraft operators for hazard mitigation nd mission planning purposes

How Using cometary ephemerides, ejection is simulated in 1 hour time steps while the comet is within $2.5 \mathrm{AU}$ of the Sun. A variable step integrator is used to integrate particle position and velocity forward in time. Nodal crossing times are recorded, as are various other parameters for particles approaching Earth during specified time periods.

Immediate Aim To investigate the effect recent updates to the model have on various Leonid and Perseid streams (in regards to peak time and duration); to show the results of modeling the

Draconids and Aurigids with the MSFC model for the first time.

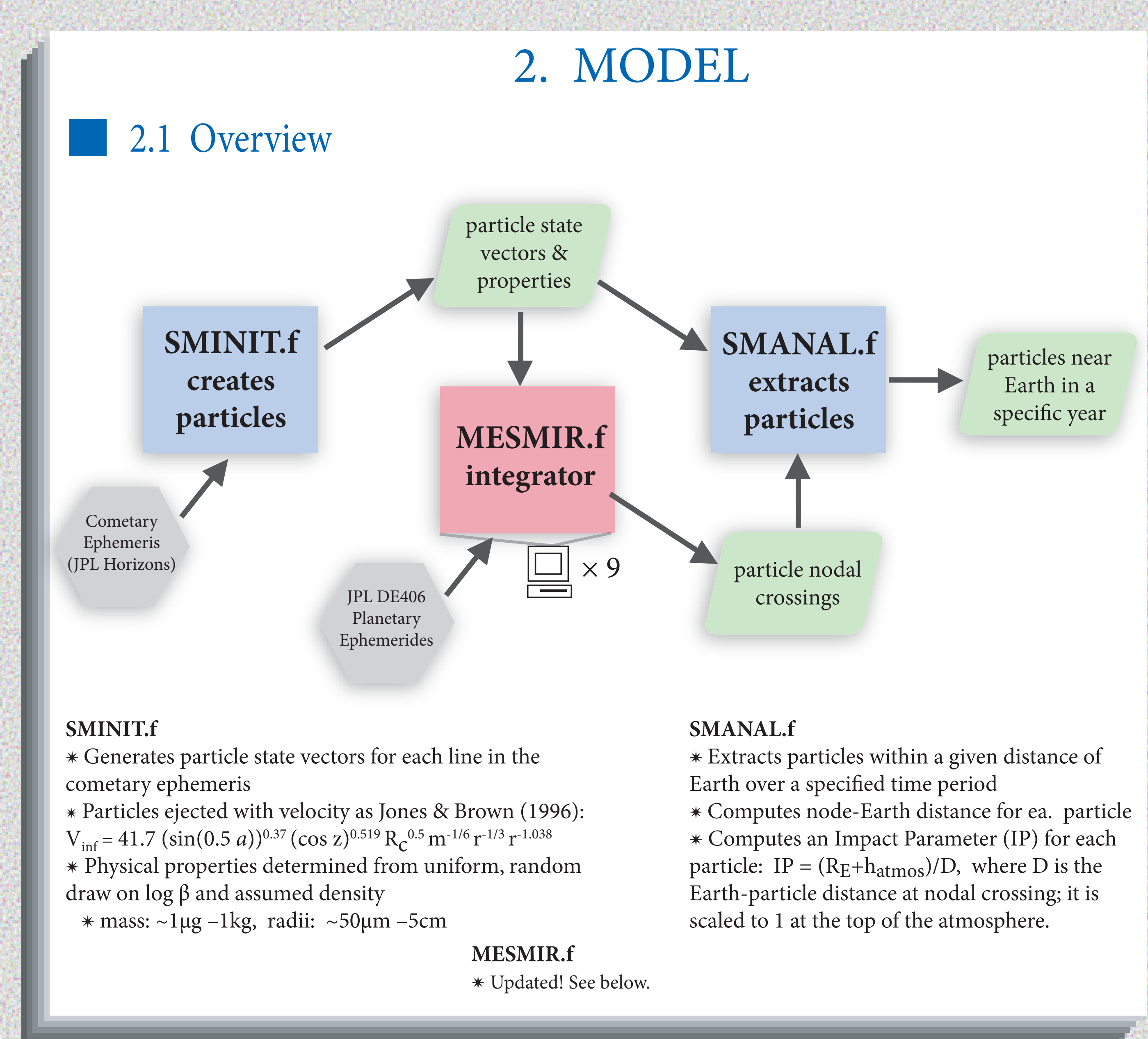

2.2 MESMIR Updates

* RK4 integrator

* Mercury included in mass of

the Sun

* Looked at perturbations from

Earth-Moon barycenter

* Effects from 7 planets; Pluto

not counted

* Interpolated planet position

(with a cubic spline) from a

look-up table of planetary posi-

tions given every day from 1000

to $2150 \mathrm{CE}$

* Only had 1 PR drag term; had a

units mismatch

* No general relativistic correc-

tion in force function * RADAU15 integrator * Mercury resolved as separate body

* Perturbations from Earth and Moon treated separately * Effects from 8 planets; Pluto counted

* Interpolates planet position (with Chebychev polynomials) from JPL Horizons from 3000 BCE to $3000 \mathrm{CE}$

* Two PR drag terms; mismatch corrected included in force function
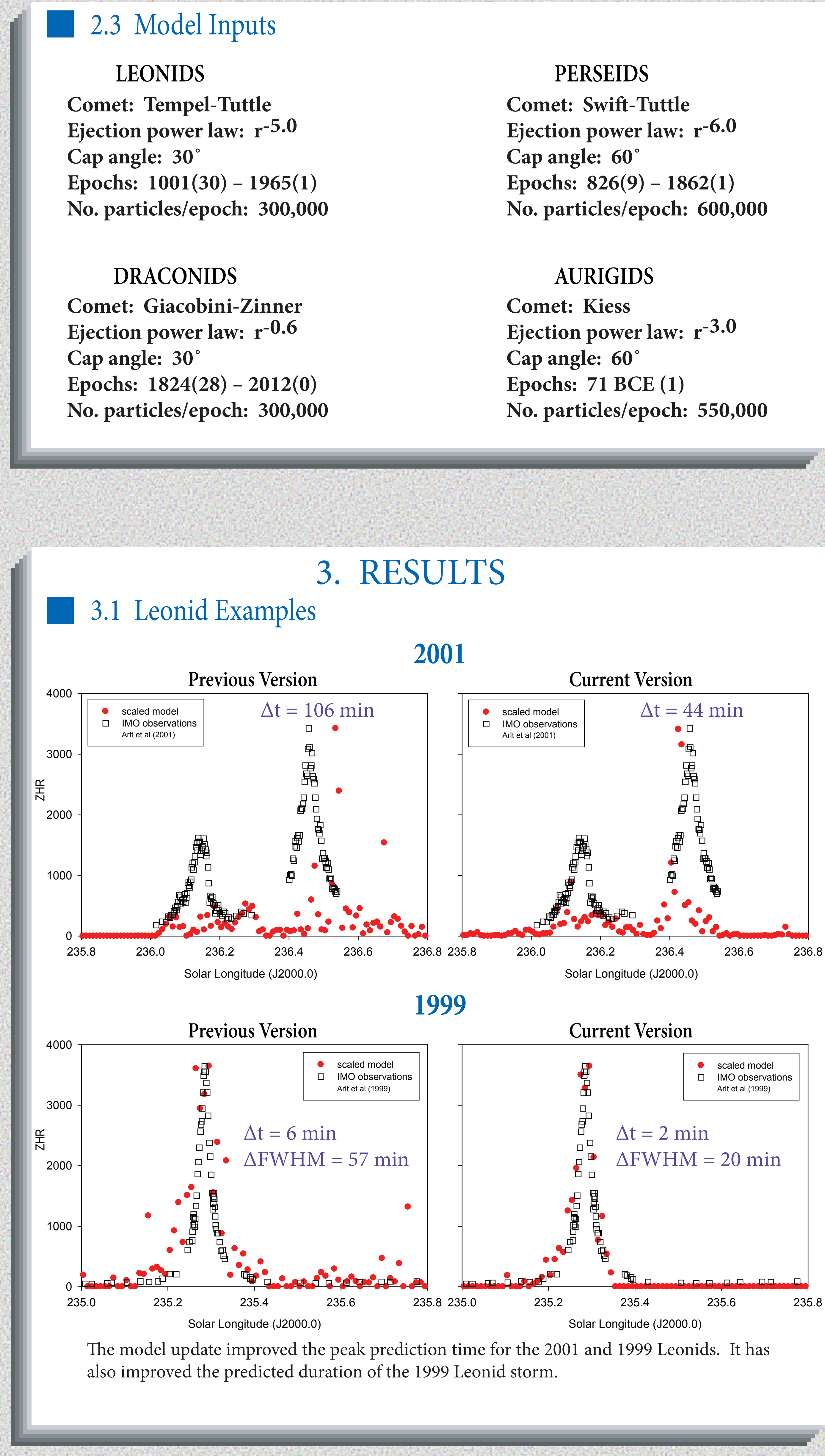
from binary files \& subroutines

* General relativistic correction
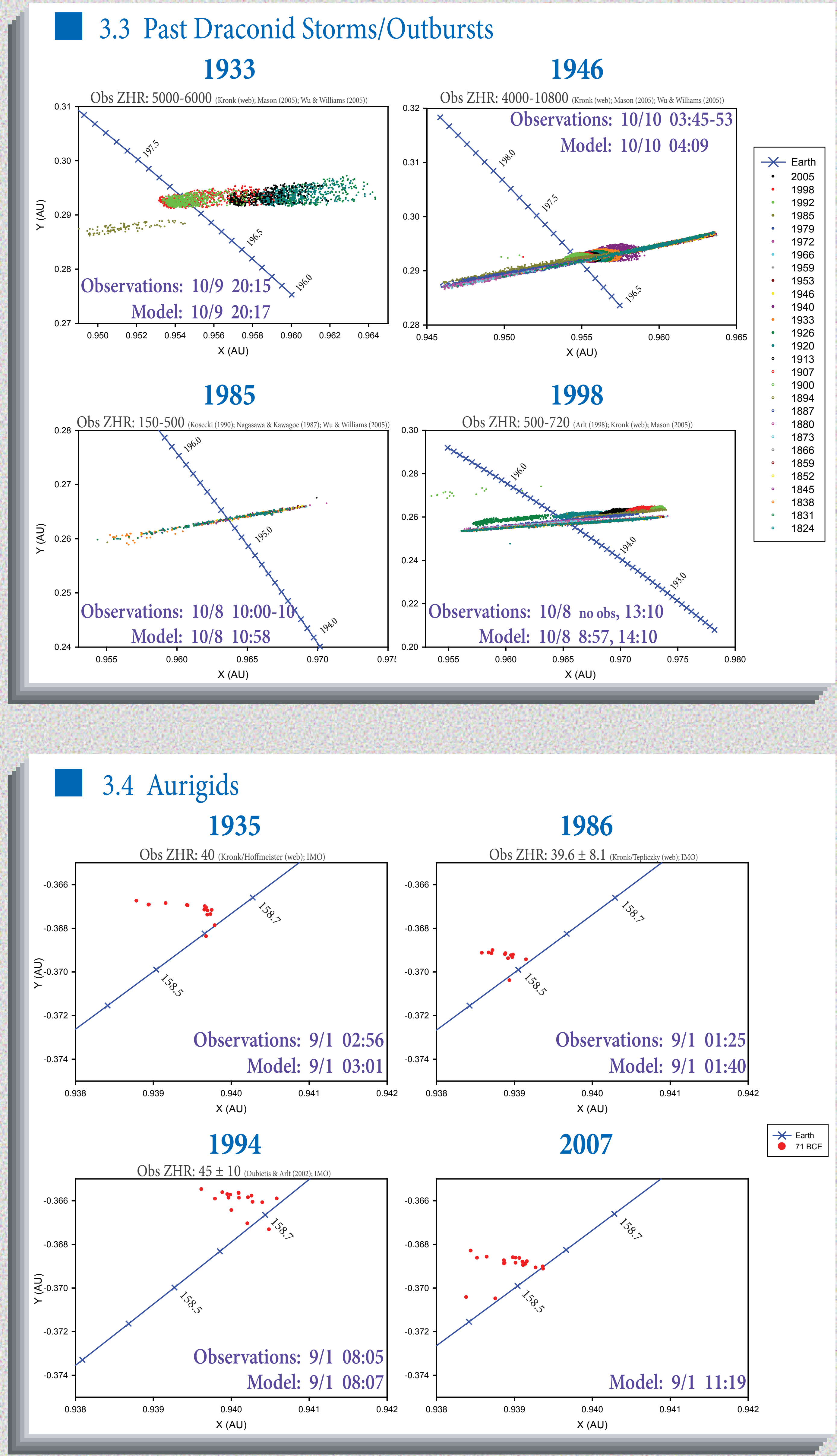

\section{SUMMARY}

Updates to the MSFC Meteoroid Stream Model better constrain the peak time and duration of the Leonid meteor showers. Improvements to the recent Perseid outbursts were not seen The MSFC model was put to the task of modeling both the Draconids and Aurigids for the first time this year. The Draconid outburst/storm peak predictions were surprisingly good, even though the IP approach for Giacobini-Zinner is not thought to be valid. There was some concent about the Aurigids this year, but according to the node, the 2007 Aurigids will be on par with showers seen in 1935, 1986, and 1994: ZHR in the 40-50s (no storm).

\section{ACKNOWLEDGEMENTS}

This work was supported by NASA contract NNM04AA02C. The authors also wish to acknowledge the IMO; a great number of their compiled observations were used as bases of comparison. Thanks also should go to Wade Batts, whose help reducing the new MESMIR's run-time was invaluable, and to Jeremie Vaubaillon, whose help and advice was greatly ap - 